\title{
Lethal Mutagenesis in Viruses and Its Effects
}

\author{
Yasemin ÇİÇEK YILDIZ ${ }^{1 a^{*}}$ Ahmed Eisa ELHAG ${ }^{2 b}$ Zafer YAZICI ${ }^{2 c}$ \\ ${ }^{1}$ Samsun Veteriner Kontrol Ensititüsü Müdürlüğü Atakum, Samsun, TÜRKIYE \\ ${ }^{2}$ Ondokuz Mayıs Üniversitesi Veteriner Fakültesi Samsun, TÜRKIYE
}

${ }^{a}$ https://orcid.org/0000-0002-2850-9912

*Sorumlu yazar: cicekyasemin@gmail.com

\section{A B S T R A C T}

Viruses are biological systems with wide variations in mutation rates. Viruses with highly accurate and conforming transcriptases have relatively low mutation rates. In contrast, viruses with high aberration transcriptases show high mutation rates, and high mutation rates can lead to higher genetic diversity. Viruses cannot be increased further without sacrificing by viral consistency according to the adaptive landscapes. A mutation can be defined as permanent changes that occur in the nucleotide sequence or the structure of nucleotides, often resulting in genetic material changes and structural disruption, thus, affect the polypeptide synthesis. Mutations can be created spontaneously or by physicalchemical properties. The nucleic acid mutations in viruses also determine their genome characteristics. Lethal mutagenesis is a broad-spectrum antiviral strategy that takes advantage of the high mutation rate and low mutation tolerance of many RNA viruses. Mutagenic drugs employ this strategy to increase the mutation rate of the virus, thus, leading a large number of mutations in the viral population, either lethal or highly harmful for continuesity of replication. Such an example Acyclovir (ACV), which is used for effective treatment in herpes simplex virus infections, works by blocking the thymidine kinase enzyme of the virus, only by entering the virus-infected cells. however, the virus developed resistance to this mechanism by generating mutant strains lacking thymidine kinase enzyme. In determining virus mutations, comparison with wild type is made phenotypically, but since it is very difficult to make this comparison in a genome that is found to be mutated frequently, making viral genome sequences has become a more effective method.
\end{abstract}

ARTICLE INFO

Review article

Received: 27.08 .2021

Accepted: 09/11/2021

Keywords:

Mutagenesis, Virus, Chemical 


\section{INTRODUCTION}

A mutation is a replication of the genome due to errors made by the polymerase enzymes that replicate DNA or RNA. Mutations are not limited to just replication processes as they may result from the editing of genetic material (induced mutations) or spontaneous nucleic acid damage ((Sanjuán and Domingo-Calap 2016; Sanjuán 2010; Yeşilbağ 2002; Drake and Holland 1999).

Mutations can be created spontaneously or by physically-chemically means. The nucleic acid mutations in viruses may determine their genome characteristics. Viruses with highly accurate and compatible transcriptases have relatively low mutation rates (for example, poliovirus RNA-dependent RNA polymerase/transcriptase) (Duffy, 2018). Whereas viruses with high deviating transcriptases show high mutation rates (influenza viruses) (Sanjua'n and Domingo-Calap 2016; Sanjuán 2010; Yeşilbağ 2002).

The mutation rate of an organism is defined as the probability of a change in genetic information that will be passed on to the next generation (Acevedo et al. 2014; Drake and Holland 1999). One generation term in viruses is often defined as the cycle of cell infection that involves cell surface binding, entry, gene expression, replication, encapsulation, and release of infectious particles ( Yeşilbağ, 2002). The mutation rate should not be confused with the frequency with which mutations are found in a particular viral population. The frequency with which mutations are found in the viral population is a measure of genetic variation that depends on other several processes, such as natural selection, random genetic drift, recombination (Tang et al. 2019; Sanjua'n and Domingo-Calap 2016). High mutation rates lead to higher genetic diversity, it is not possible to deduce the mutation rates directly from the observed population mutation frequencies (Zinshteyn and Nishikura 2009; Yang et al. 2005).

Whereas genetic variation depends on multiple factors, particular attention should be paid to the rate of mutation because it is the source of genetic variation (Drake and Holland 1999). Likewise, mutation rates should not be confused with molecular evolution rates (Rotem et al. 2018). Neutral molecular evolution theory shows a linear relationship between these two ratios, however, while mutation is a biochemical/genetic process, molecular evolution refers to the fixation of new alleles in populations (Domingo-Calap 2016; Sanjuán 2010; Zinshteyn and Nishikura 2009; Yang et al. 2005; Drake and Holland 1999).

\section{Viral and Lethal Mutagenesis}

The knowledge of viral mutation rates will help to understand and manage drug resistance, anti-viral immunity, vaccination strategies, viral pathogenesis and processes of new diseases emergence. Viral mutation rates are modulated at different levels according to many factors including polymerase activity, cellular microenvironment, replication mechanisms, post-replication proofreading mechanisms (Domingo-Calap et al. 2012). A large number of mutations can be introduced by cell-encoded cytidine/adenine deaminases, as well as some virus-encoded diversity generating functions (Zinshteyn and Nishikura 2009; Yang et al. 2005).

Viruses are biological systems with wide variations in mutation rates, because there are great differences between RNA and DNA viruses. While the polymerase enzyme contributes to viral mutation rates, not only through causing errors, but also allows the ability of self correction by DNA post-replication repair (profeeding mechanism) (Tang et al. 2019; Domingo-Calap et al. 2012). Single-stranded DNA viruses are the fastest mutating DNA viruses, with mutation rates per nucleotide that could close to some RNA viruses, but chemical and lethal mutagenesis have been less studied on these types of viruses (Sanjuán, 2010; Graci et al. 2007). Also, other sources of mutation include cellular enzymes, spontaneous nucleic acid damage, and special genetic elements that found within some viral genomes whose specific function is to produce new mutations (Acevedo et al. 2014; Pfeiffer and Kirkegaard 2003). However, viral genetic diversity determined by mutation rates has an important effect on the design of antiviral strategies. (Domingo-Calap 2016; Sanjuán 2010; Zinshteyn and Nishikura 2009; Yang et al. 2005; Drake and Holland 1999).

Reorganization studies of viruses are a rapidly expanding and developing area of research. The most studied part was the adenosine deaminases (ADAR) that target dsRNA sites and convert adenosine (A) to inosine (I) and cause a change from A to guanosine $(\mathrm{G})$ after second strand synthesis (Zinshteyn and Nishikura 2009). ADARs target mRNAs, the transposable elements and genomes of RNA viruses. In mammals, A to G mutations thought to be caused by ADARs in several viral families that have been studied (Zinshteyn and Nishikura, 2009; Yang et al. 2005).

The distinguishing feature of RNA viruses is their high mutation rates due to RNA polymerases replication process, which lack redaction mechanisms (Drake and Holland 1999). An RNA virus population can form a complex population structure consisting of a main sequence with closely related genetic variants, and this is often referred to as a quasispecies or mutant spectrum (Acevedo et al. 2014; Sanjuán 2010; Drake and Holland 1999).

It is generally accepted that mutation rates cannot be increased further without sacrificing by viral consistency according to the adaptive landscapes. Supporting this view, serial passages in the presence of nucleoside analogues or 
other mutagens have been shown to cause severe losses or even population extinction through lethal mutagenesis in a variety of RNA viruses, especially arenaviruses, lentiviruses, enteroviruses, and hantaviruses (Bassi et al. 2018; Acevedo et al. 2014; Severson et al. 2003). This has led to the suggestion that lethal mutagenesis can be used as a therapeutic strategy against RNA viruses (Rotem et al. 2018; Acevedo et al. 2014; Morfin and Thouvenot, 2002).

Broad-spectrum antiviral developing is used lethal mutagenesis, that takes into account the high mutation rate and low mutation tolerance of many RNA viruses. This approach uses mutagenic drugs to increase the mutation rate of the virus, thus leading the viral population to a large number of mutations that either lethal or highly harmful for continuesity of replication (Graci et al.2008; Graci et al.2007; Severson et al. 2003). Lethal mutagenesis has been applied to many RNA viruses, and nucleoside base analogs are most commonly used. (Bassi et al. 2018; Pauly and Lauring 2015; Domingo-Calap et al. 2012).

Lethal mutagenesis is usually detected by a concentration-dependent decrease in infectious viral titer, an increase in the frequency of viral mutations, a concentration-dependent decrease in the specific infectivity of the viral population, and the ability to destroy the viral population over multiple replication cycles in the presence of the mutagenic drug (Pauly and Lauring 2015; Bull et al. 2007).

Selected nucleoside analogues may develop an increase in virus replication and tolerance to mutation by keeping them away from the polymerase active site. In most cases, the mutagenic activity of nucleoside analogs can be attributed to the incorrect incorporation of triphosphate forms into replicated genomes by the viral RdRp (polymerase) (Severson et al. 2003; Sierra et al. 2000). The structure of the nucleoside analog, nucleoside base properties and RNA polarity determine the classes of mutations that ocur (Graci et al. 2007; Pfeiffer and Kirkegaard 2003). Ribavirin, a guanosine analog, causes an increase in both C-to-U and G-to-A transitional mutations. 5-fluorouracil, the urinidine analog, leads to $A$ to $\mathrm{G}$ mutations and $\mathrm{U}$ dimers. Also, 5-azacitidine, a cytidine analog, can induce both $\mathrm{C}$ to $\mathrm{G}$ and $\mathrm{G}$ to $\mathrm{C}$ transversion mutations through a pyrimidine ring opening mechanism that allows it to base pair with cytosine (Bassi et al. 2018; Graci et al. 2006). Ribavirin also has additional mechanisms that may play a role in its antiviral activity. In host cells, it alters GTP pool concentrations by inhibiting IMP dehydrogenase (IMPDH) (Graci et al. 2006; Severson et al. 2003; Sierra et al. 2000). Among other effects, it has been shown to be able to induce direct inhibition of influenza virus RdRp and transcription of viral RNA. Also, some studies show that ribavirin affects in vivo inflammatory and T cell responses (Tang et al. 2019; Pauly and Lauring 2015; Domingo-Calap et al. 2012; Graci et al. 2008; Graci and Cameron 2006; Graci et al. 2007; Severson et al. 2003).

The mutation site created by a virus is an important finding of the genetic background that affects the efficiency of lethal mutagen. It is known that during influenza virus replication, negative polarity viral RNA is transcribed at 10 to 100 times higher levels than positive cRNA (Domingo-Calap 2016; Sanjuán R 2010; Drake and Holland 1999). This indicates that for a given nucleotide position, the nucleoside analog is more likely to be involved in the negative strand. In some studies, it has been determined that the influenza virus RdRp has a particularly strong tendency to transitional mutations from $\mathrm{A}$ to $\mathrm{G}$ and $\mathrm{U}$ to $\mathrm{C}$, and this mutational tendency allows influenza virus populations to have a different sequence domain through this type of mutation. Which can be explored comprehensively (Pauly and Lauring 2015; Zinshteyn and Nishikura 2009). However, it has also been that it can confer a certain level of genetic resistance to the harmful effects of A-to-G and U-to-C transmission. Therefore, mutagens that induce the same type of mutations of the influenza virus may be less effective at inducing lethal mutagenesis (Fonville et al. 2014). In a different study, it was determined that using 5-fluorouracil, can mimics the two most common types of mutations made by influenza virus $\mathrm{RdRp}$ and causes a decrease in the specific infectivity of RdRp. These results show that to reach maximum effect, a lethal mutagen must force a viral population to discover regions of the sequence domain that are not normally accessed under normal replication conditions (Pauly and Lauring 2015).

In studies with flaviviruses viruses, Zika virus (ZIKV) has an important place in studies. Because flaviviruses pose a public health threat due to their rapid spread to geographically temperate climates and the increasing in their number of pathogens. There are limited data on the sensitivity of ZIKV to lethal mutagenesis. A studies showed that ZIKV was susceptible to three ribonucleosis (favipiravir, ribavirin and 5-fluorouracil), which showed also mutagenic activity against other RNA viruses, while remaining unaffected by the mutagenic deoxyribonucleoside. The effect of these mutagens resulted in rapid elimination of infectivity with ZIKV upon serial cell culture passages. However, it has been found to be relatively sensitive in serial passages in the presence of purine analogs (favipiravir and ribavirin). With these results, it was predicted that regulation of flavivirus replication and antiviral treatments based on lethal mutagenesis could improve (Bassi et al. 2018; Severson et al. 2003).

Studies have shown that poliovirus, foot-and-mouth disease virus and chikungunya virus, mutagen resistant variants, recovered after 14 passages at non-lethal drug concentrations (Graci et al. 2008; Graci et al. 2007; Sierra et al. 2007; Sierra et al. 2000). The RdRps of these resistant viruses have replication fidelity phenotypes that make them less susceptible to mutagenesis by their nucleoside analogues. Population genetic theory suggests that higher mutation rates 
will select viruses that are more tolerant or that don't mutate. This mutagenic mechanism of drug tolerance has been recently described in vesicular stomatitis virus populations undergoing 5-fluorouracil antiviral therapy and coxsachia virus populations undergoing ribavirin antiviral therapy (Tang et al. 2019; Rotem et al. 2018; Pauly and Lauring 2015; Sanjuán 2010; Yang et al. 2005; Severson et al. 2003).

\section{CONCLUSION}

As a conclusion viruses make up the majority of mutations in nature. Viruses with high mutation rates tend to evade immunity more effectively (Sanjua'n and Domingo-Calap 2016; Fonville et al. 2014; Sanjuán 2010). Therefore, the mechanisms of action of chemical components that support lethal mutagenesis need to be investigated further.

\section{CONFLICT OF INTERESTS}

The authors declare that there is no conflict of interest

\section{AUTHORS CONTRIBUTION}

All authors have participated in drafting the article and approved the final version.

\section{REFERENCES}

Acevedo A, Brodsky L, Andino R (2014). Mutational and fitness landscapes of an RNA virus revealed through population sequencing. Nature 505, 686-690.

Bassi M R, Sempere R N, Meyn P, Polacek C, Arias A (2018). Extinction of Zika Virus and Usutu Virus by Lethal Mutagenesis Reveals Different Patterns of Sensitivity to Three Mutagenic Drugs Antimicrob Agents Chemother,27;62(9):e00380-18.

Bull J J, Sanjuán R, Wilke O C (2007). Theory of lethal mutagenesis for viruses. J. Virol. 81:2930-2939.

Domingo-Calap P, Pereira-Gómez M, Sanjuán R (2012). Nucleoside Analogue Mutagenesis of a Single-Stranded DNA Virus: Evolution and Resistance J Virol, 86(18):9640-6.

Drake J W, Holland J J (1999). Mutation rates among RNA viruses. PNAS,23, 96(24) 13910-13913 Duffy S (2018). Why are RNA virus mutation rates so damn high. PLoS Biol 16:8.

Fonville J M, Wilks S H, James S L, Fox A, Ventresca M, Aban M, Xue L, Jones T C, Le N M H, Pham Q T (2014). Antibody landscapes after influenza virus infection or vaccination. Science 346(6212): 996-1000.

Graci J D, Harki D A, Korneeva V S, Edathil J P, Too K, Franco D, Smidansky E D, Paul AV, Peterson B R, Brown D M, Loakes D, Cameron C E (2007). Lethal mutagenesis of poliovirus mediated by a mutagenic pyrimidine analogue. J. Virol. 81:11256-11266.

Graci J D, Too K, Smidansky E D, Edathil J P, Barr E W, Harki D A, Galarraga J E, Bollinger J M Jr, Peterson B R, Loakes D, Brown D M, Cameron C E (2008). Lethal mutagenesis of picornaviruses with N-6- modified purine nucleoside analogues. Antimicrob. Agents Chemother. 52:971-979.

Graci J D, Cameron C E (2006). Mechanisms of action of ribavirin against distinct viruses. Rev. Med. Virol. 16:37-48.

Morfin F, Thouvenot D (2003). Herpes simplex virus resistance to antiviral drugs. Journal of Clinical Virology 26:2937.

Pauly M D, Lauring A S (2015). Effective Lethal Mutagenesis of Influenza Virus by Three Nucleoside Analogs J Virol,89(7): 3584-97.

Pfeiffer J K, Kirkegaard K (2003). A single mutation in poliovirus RNA-dependent RNA polymerase confers resistance to mutagenic nucleotide analogs via increased fidelity. Proc. Natl. Acad. Sci. USA 100:7289-7294.

Rotem A, Serohijos A W R, Chang C B, Wolfe J T, Fischer A E, Mehoke T S, Zhang H, Tao Y, Ung W L, Choi J M, Rodrigues, J V, Kolawole A O, Koehler S A, Wu S, Thielen P M, Cui N, Demirev P A, Giacobbi N S, Julian T R, Schwab K, Lin J S, Smith T J, Pipas J M, Wobus C E, Feldman A B, Weitz D A, Shakhnovich E I (2018). Evolution on the Biophysical Fitness Landscape of an RNA Virus Mol. Biol. Evol. 35(10):2390-2400

Sanjua'n R, Domingo-Calap P (2016). Mechanisms of viral mutation Cell. Mol. Life Sci 73: 4433-4448.

Sanjuán R (2010). Mutational fitness effects in RNA and single-stranded DNA viruses: common patterns revealed by site-directed mutagenesis studies. Philos Trans R Soc Lond B Biol Sci. 27;365(1548):1975-82

Severson W E, Schmaljohn C S, Javadian A, Jonsson C B (2003). Ribavirin causes error catastrophe during Hantaan virus replication. J. Virol. 77:481-488

Sierra M, Airaksinen A, González-López C, Agudo R, Arias A, Domingo E (2007). Foot-and-mouth disease virus mutant with decreased sensitivity to ribavirin: implications for error catastrophe. J. Virol. 81: 2012-2024.

Sierra S, Da'vila M, Lowenstein P R, Domingo E (2000). Response of foot-and-mouth disease virus to increased mutagenesis: influence of viral load and fitness in loss of infectivity. J. Virol. 74:8316-8323 
Çiçek Yıldiz et al. / Manas Journal of Agriculture Veterinary and Life Sciences 11 (2) (2021) 194-198

Tang J, Brixel R, Brune W (2019).Copy-Paste Mutagenesis: A Method for Large-Scale Alteration of Viral Genomes Int J Mol Sci. 20(4): 913.

Yang W, Wang Q, Howell KL, Lee J T, Cho D S (2005).ADAR1 RNA deaminase limits short interfering RNA efficacy in mammalian cells. J Biol Chem. 280:3946-3953.

Yeşilbağ K (2002). Mutational Changes and Importance in Veterinary Virology. Uludag Univ. J. Fac. Vet. Med. 21; 125-131

Zinshteyn B, Nishikura K (2009).Adenosine-to-inosine RNA editing Wiley Interdiscip Rev Syst Biol Med.1(2): 202209 